\title{
Moyamoya disease manifested as multiple simultaneous intracerebral hemorrhages: A case report and literature review
}

\author{
JINLU YU ${ }^{1}$, YONGJIE YUAN ${ }^{1}$, WEI LI $^{2}$ and $\mathrm{KAN} \mathrm{XU}^{1}$ \\ ${ }^{1}$ Department of Neurosurgery, The First Hospital of Jilin University, Changchun, Jilin 130021; \\ ${ }^{2}$ Department of Neurosurgery, The Second Affiliated Hospital of Xingtai Medical College, Xingtai, Hebei 054000, P.R. China
}

Received May 22, 2015; Accepted June 2, 2016

DOI: $10.3892 /$ etm.2016.3477

\begin{abstract}
Multiple simultaneous intracerebral hemorrhages (MSIH) caused by Moyamoya disease (MMD) is extremely rare. To date, the clinical manifestations, imaging characteristics and mechanism of MMD-induced MSIH have not yet been elucidated. In order to improve the understanding on such cases, the present study described a rare case of MSIH caused by MMD. A 40-year-old female patient with no history of hypertension or diabetes mellitus experienced a sudden headache followed by coma. Cranial computed tomography (CT) examination revealed $\mathrm{MSIH}$ in the left frontal area, temporal lobe and basal ganglia. CT angiography and digital subtraction angiography examinations revealed typical characteristics of MMD. Subsequent to excluding disorders of the blood system and blood coagulation, we concluded that the present case of MSIH was caused by MMD. Hematoma evacuation and decompressive craniectomy were performed with satisfactory results. In addition, after reviewing previous MSIH cases in the literature, potential mechanisms of MMD-mediated MSIH were considered. In conclusion, MMD should be considered as a possible cause of MSIH during diagnosis and treatment. MMD can lead to pathological changes in the fragility of small arteries; therefore, rupture and hemorrhage at one site may induce a transient increase in blood pressure, causing the rupture of small arteries at other sites, and thus leading to MSIH. Hematoma evacuation and decompression should be conducted in selective cases of MMD-induced MSIH in order to achieve a good prognosis.
\end{abstract}

\section{Introduction}

Multiple simultaneous intracerebral hemorrhages (MSIH) is rare phenomenon, accounting for $\sim 3 \%$ of all intracranial hemorrhage events (1). MSIH remains intriguing regarding

Correspondence to: Dr Kan Xu, Department of Neurosurgery, The First Hospital of Jilin University, 71 Xinmin Avenue, Changchun, Jilin 130021, P.R. China

E-mail: jlyu@jlu.edu.cn

Key words: moyamoya disease, multiple, intracranial hemorrhage the causes and MMD can be caused by a variety of factors, including hypertension, cerebral vasculitis, sympathetic nerve stimulants, drugs that affect blood coagulation, brain tumors, cerebral amyloidosis, venous sinus thrombosis and coagulation disorders, in addition to other unexplained factors $(2,3)$. The treatment for MSIH mainly consists of releasing the intracranial pressure caused by the hematoma, identifying the cause of the original hemorrhage and then providing surgical treatment (4). Although there are numerous studies describing MSIH in the literature, MSIH of Moyamoya disease (MMD) is rare. Kikuta et al (5) in 2008 reported a series of multiple intracerebral hemorrhages of MMD, but these hemorrhages were limited to multiple trace bleeding. However, to the best of our knowledge, no case of severe MSIH caused by MMD has been reported to date. MMD is an uncommon disease characterized by progressive occlusion of the terminal portion of the internal carotid artery and its main branches within the circle of Willis. This occlusion results in the formation of a fine vascular network at the base of the brain. These MMD vessles are fragile and easy to rupture (6); therefore, MSIH in MMD is possible. Intracranial hemorrhage in MMD is not easy to treat (7), so MSIH would be more difficult to treat.

The present study reports a rare case of severe MSIH caused by MMD, which was treated with hematoma evacuation and decompressive craniectomy, resulting in good recovery. In addition to the case report, the mechanism of MMD-induced MSIH is also discussed.

\section{Case report}

Written informed consent was obtained from the patient. A 40 -year-old female patient with no history of hypertension and diabetes mellitus was admitted the First Hospital of Jilin University (Changchun, China) in June 2015 due to presentation of sudden severe headache and vomiting followed by gradual coma for $2 \mathrm{~h}$. Physical examinations revealed stable vital signs with a blood pressure of $140 / 90 \mathrm{mmHg}$, and the neurological examination showed minor coma (Glasgow Coma Scale score, 11) (8), right hemiplegia, degree 3 muscle strength, positive Babinski sign, and stiffness in the neck with Kernig's sign (9). Laboratory tests indicated normal blood coagulation and platelet counts. Cranial computed tomography (CT) revealed multiple irregular high density zones in the left basal ganglia, posterior temporal lobe and frontal lobe, with 
A

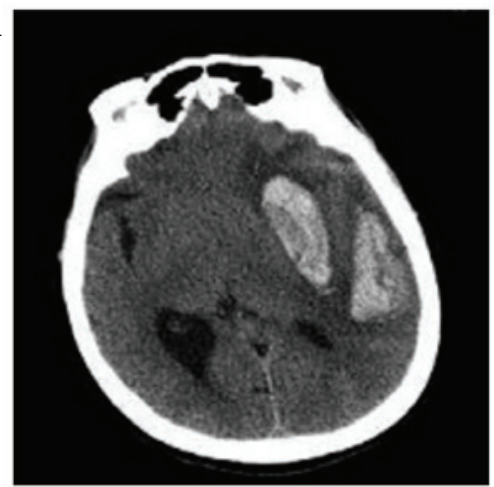

C

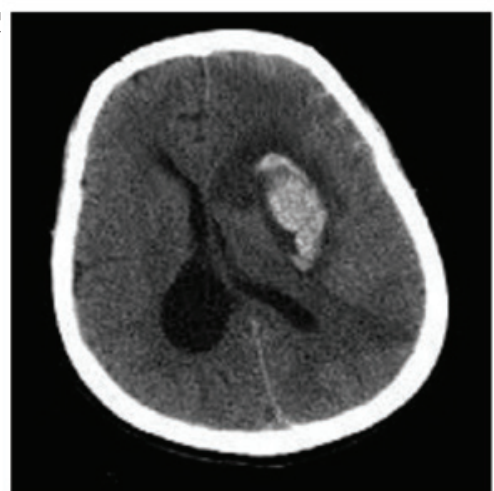

E

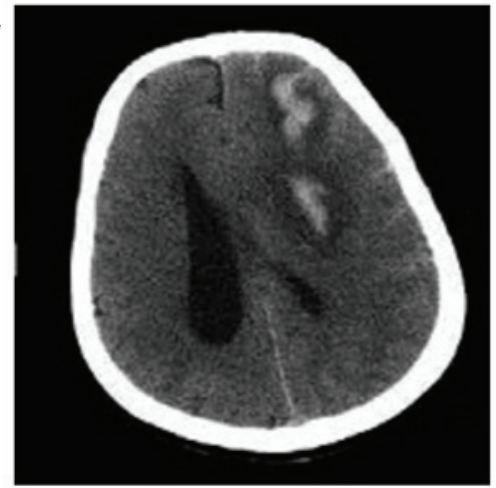

B

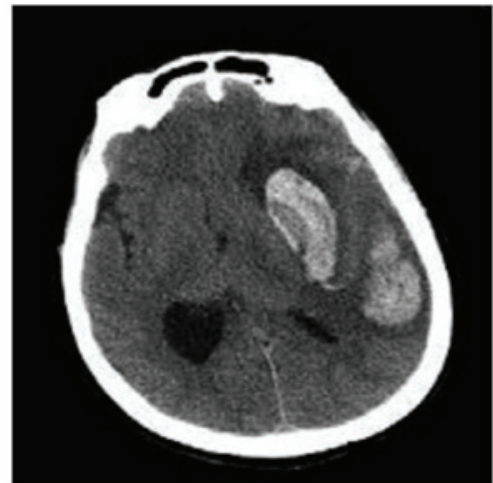

D

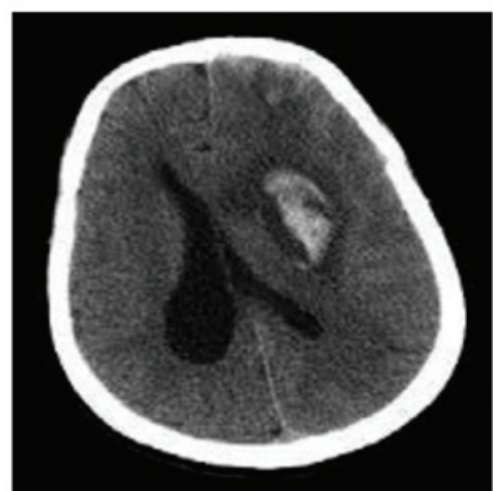

F

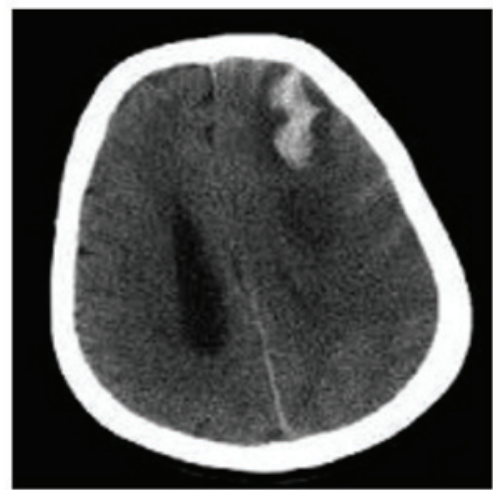

Figure 1. Preoperative cranial computed tomography scans showing multiple intracranial hemorrhages in sections of the (A and B) basal ganglia, (C and D) lateral ventricle body and (E and F) lateral ventricle loop. Irregular high density zones in the basal ganglia, posterior temporal lobe and frontal lobe, with mild peripheral edema. The left ventricle showed deformation under compression, with the midline shifted to the right.

mild peripheral edema. The left ventricle showed deformation under compression, with the midline shifted to the right (Fig. 1). Cranial CT angiography (CTA) subsequent to admission demonstrated that the normal vascular morphology in the area of the bilateral middle cerebral artery was disrupted, with visible hematomas in the maximum intensity projection axis (Fig. 2). Based on the symptoms and the radiographic examination findings, a clear diagnosis of MMD and intracerebral MSIH was established.

An emergency hematoma evacuation and decompressive craniectomy was scheduled. To further clarify the circumstances of the intracranial arteries, preoperative digital subtraction angiography (DSA) was performed under general anesthesia in the catheterization room. The DSA results showed occlusions at the ends of the bilateral carotid arteries, which were replaced with 'smoke-like' blood vessels (Fig. 3). With the exception of these smoke-like blood vessels, no aneurysms or vascular malformations were observed in the areas of the left middle cerebral artery or anterior artery areas (Fig. 3). Hematoma evacuation and decompressive craniectomy were performed under general anesthesia in order to remove the majority of the hematoma in the left basal ganglia and posterior temporal lobe; however, the hemorrhage in the left frontal lobe was not treated as it was risky and unnecessary. Following hematoma evacuation, the temporal muscle was attached to the brain surface for fusion. A postoperative CT scan revealed that the majority of the intracranial hematoma was removed and that the ventricular size had returned to normal, with the midline in the center (Fig. 4). Postoperative nutritional support, including Nutrison Fibre $(80 \mathrm{ml} / \mathrm{h}$ intranasally; Nutrica Pharmaceutical Co., Ltd., Wuxi, China) and trimebutine maleate (400 mg once every three days; Tanabe Pharmaceutical Co., Ltd., Tianjin, China), and symptomatic treatment were provided for 1 week, and the patient gradually regained consciousness, with motor aphasia, low pressure in the left decompression window, right hemiplegia and degree 3 muscle strength. Over the 3-month 


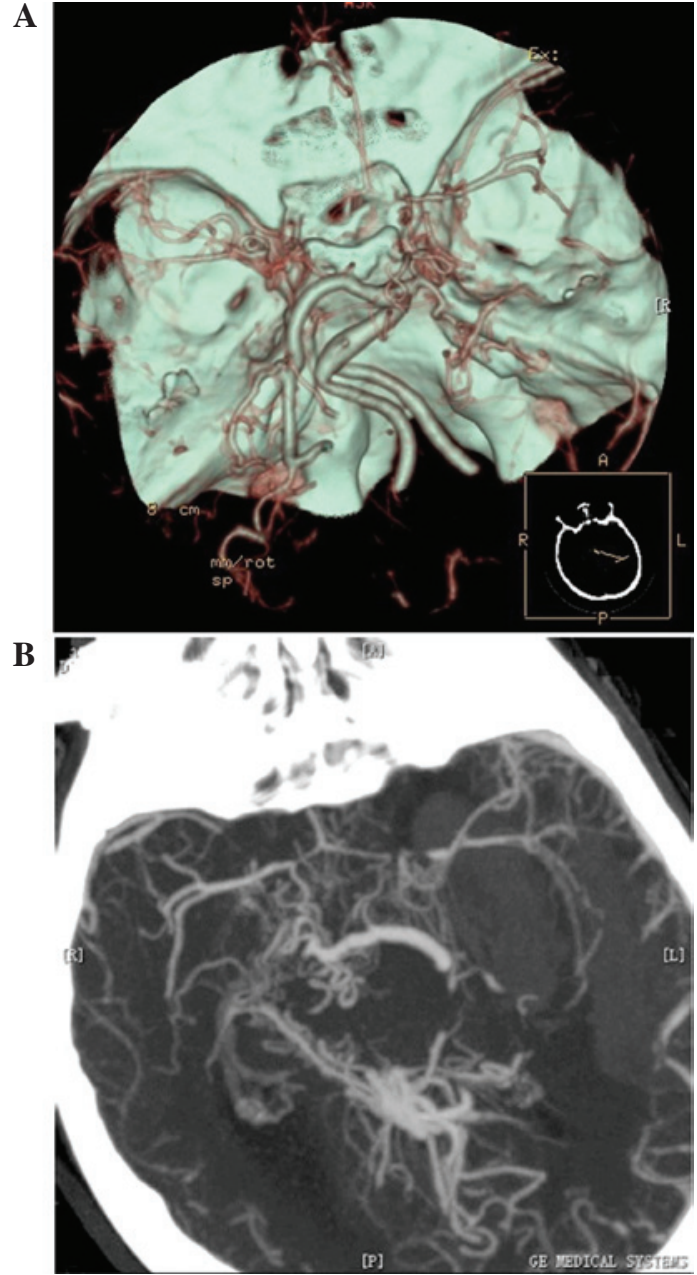

Figure 2. Cranial computed tomography angiography revealed a diagnosis of Moyamoya disease. (A) Normal vascular morphology in the area of the bilateral middle cerebral artery distribution disappeared. (B) Hematomas are observed in the area of the middle cerebral artery distribution on the left of the maximum intensity projection axis, located inside the smoke-like blood vessels.

follow-up period the patient was aphasic, with degree 4 muscle strength in her right limb, and essentially self-sufficient in her daily life. In June 2016, the patient's symptoms persisted but no hemorrhage had reoccurred, and the patient's quality of life was good.

\section{Discussion}

Intracerebral hematoma commonly occurs at a single site, although multiple hemorrhages at different sites with long intervals may also occur. By contrast, the incidence of MSIH is very rare. In 2010, Stemer et al (10) conducted a study including 522 cases of spontaneous intracerebral hemorrhage, of which 29 were MSIH patients, yielding an incidence rate for MSIH of $5.6 \%$. MSIH is defined, based on imaging examination, as multiple hemorrhages or hemorrhages at different sites occurring within $24 \mathrm{~h}$, including secondary hemorrhages with clear causes and an unexplained primary hemorrhage (10). The incidence of simple primary MSIH was estimated to be between 0.75 and $3.0 \%$, which is lower compared with the overall incidence of MSIH (10). However, the study

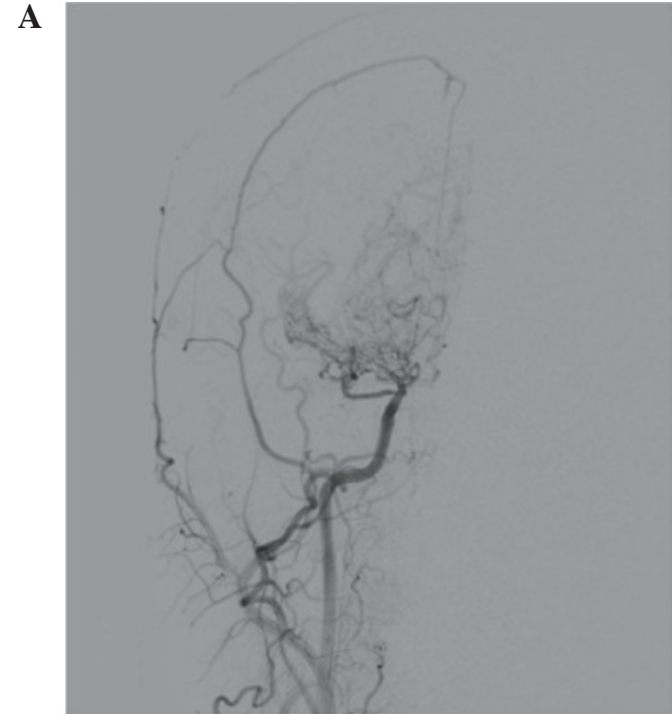

B

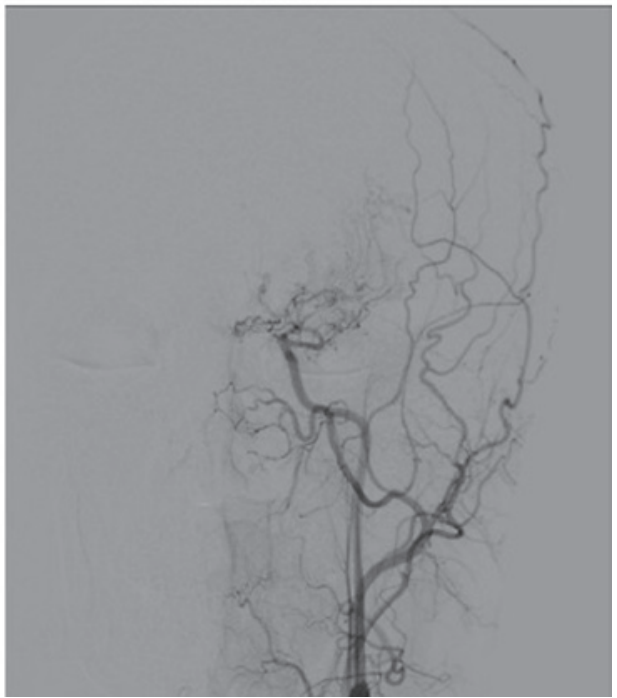

Figure 3. Cranial preoperative digital subtraction angiography revealed a diagnosis of Moyamoya disease. The ends of the bilateral carotid arteries were occluded and replaced with the smoke-like blood vessels. (A) The middle artery of the right meninges formed an intracranial collateral anastomosis at the midline. (B) No aneurysms or other vascular malformations were observed in the areas of the left middle cerebral artery or anterior artery distribution.

by Stemer and colleagues did not include MMD cases, possibly due to the majority of MMD-induced MSIH cases not being clearly identified and classified as primary MSIH. Indeed, only a limited number of MMD-induced multiple intracerebral hemorrhage cases were available in the literature. In 2014, Laiwattana et al (3) reviewed the MSIH literature published during the 60-year period between 1953 and 2013, and their results were consistent with the MSIH incidence rate reported by Stemer et al (10). In addition, the authors reviewed the literature in order to analyze the causes of secondary MSIH, although MMD was not considered as a cause of secondary MSIH in their review (3). In the present study, a case of MSIH caused by MMD was described, aiming to bring clinical attention to the fact that MMD can lead to MSIH, thereby enriching the available clinical data of MSIH.

In addition to unexplained primary factors, numerous secondary factors can cause MSIH, including arterial 


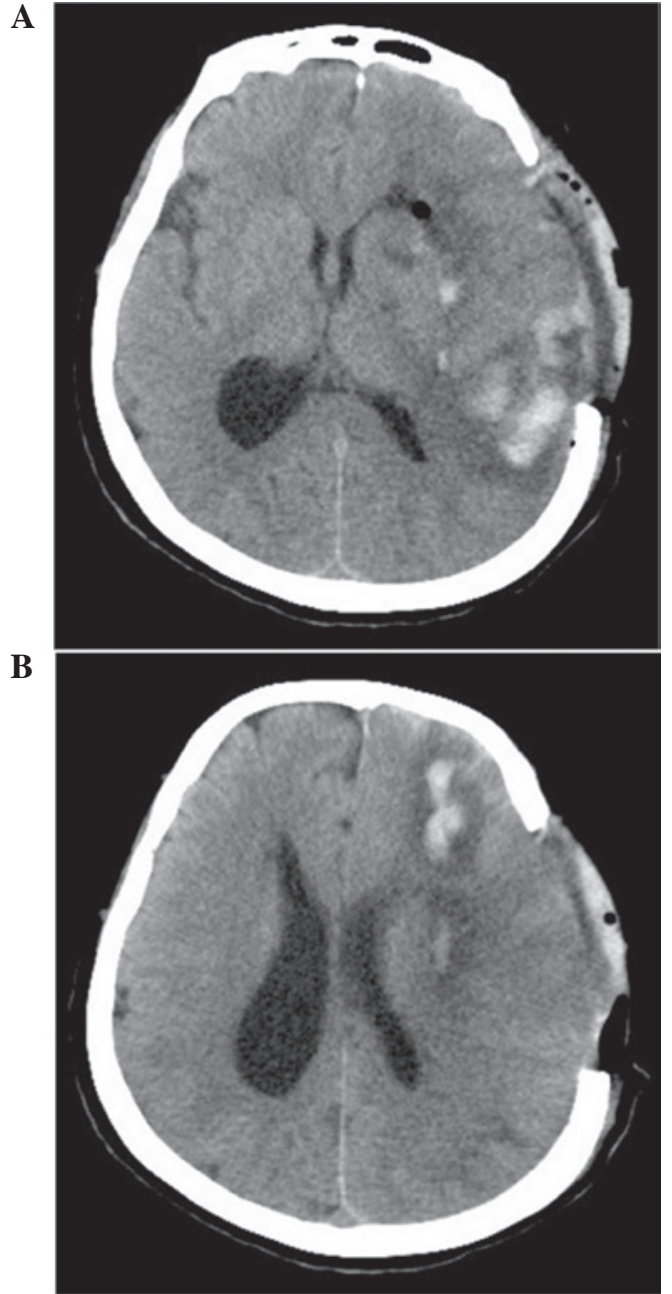

Figure 4. Postoperative computed tomography scans. (A) Basal ganglia; (B) body of lateral ventricle. The majority of the intracranial hematomas were removed, with a small amount of residual hematoma. Ventricular size returned to normal with the midline at the center.

diseases, venous drainage obstruction, parenchymal diseases (such as brain tumors and cavernous hemangioma), drug use and hematologic diseases $(4,11,12)$. However, the most common causes are arterial diseases, such as hypertension, atherosclerosis, vasculitis and cerebral amyloidosis. In 2005, Yen et al (13) reported 1,306 cases of hypertensive intracerebral hemorrhage, including 10 cases of MSIH, with an incidence rate of $\sim 0.8 \%$. MSIH caused by hypertension or by vasculitis has been reported in several other studies. For instance, in the study by McCormick and Rosenfield (2) published in 1973, 2 cases of MSIH were clearly caused by vasculitis among 144 cases of cerebral hemorrhage (2). In addition, cerebral amyloidosis may cause MSIH; for example, in 1984, Gilles et al (14) reported 11 cases of MSIH that were directly caused by cerebral amyloidosis. MSIH caused by arterial disease most likely occurs due to multiple ruptures in the diseased vessels, induced by hypertension from the original arterial disease. Alternatively, ruptures and hemorrhages in a diseased cerebral vessel may lead to a transient increase in blood pressure, thereby inducing hemorrhages at other sites in the brain, resulting in MSIH $(15,16)$.

MMD is an arterial brain disease that is characterized by occlusions at the end of the intracranial and carotid arteries.
Normal blood vessels disappear and are replaced by 'smoke-like' blood vessel hyperplasia, mainly from expanded and distorted lenticulostriate and thalamoperforate arteries (17). In these abnormal small arteries, internal elastic layer fracture, medial fibrosis and local expansion to form small aneurysms may occur $(18,19)$. Theoretically, these small diseased arteries are able to cause MSIH through hypertension, or through hemorrhage at one site that can lead to a transient increase in blood pressure and induce hemorrhages at other sites. However, MSIH caused by MMD has been rarely reported, and the few published studies were limited to multiple trace bleeding (20). For instance, the study of Kikuta et al (5) in 2008 investigated 50 cases of MMD using 3T magnetic resonance imaging (MRI), and identified 11 cases of multiple trace bleeding, which were caused due to small diseased arteries. This situation is also a risk factor for future severe cerebral hemorrhage. By contrast, MMD-induced MSIH has not been previously described, and was first reported in the present study. The cranial CT performed in the present study demonstrated no intracranial malacia, which may occasionally form subsequent to hemorrhage. One of the pathological changes caused by MMD is that certain small arteries become fragible, which manifests as internal elastic layer fracture and medial fibrosis. The rupturing and hemorrhaging of a diseased cerebral vessel may lead to a secondary transient increase in blood pressure, thereby inducing hemorrhages at other sites in the brain and resulting in MSIH.

Following MSIH, the underlying cause must be identified in order to develop an effective treatment plan. The causes of MSIH include arterial diseases, clogging of venous drainage, parenchymal diseases, drug use and hematologic diseases (3). Therefore, specific examinations can be performed, including the following: CTA, MRA or DSA to examine the arteries; CTV, MRV, or DSA to examine the intracranial veins; CT and MRI for brain parenchymal lesions; and routine blood tests for determination of platelet count, platelet function, coagulation function and blood cell sedimentation rate, in order to identify hematologic diseases (4). In addition, the medical history of the patient, including hypertension, diabetes and drug use involving sympathetic stimulants and anticoagulants, is also important. However, MMD diagnoses are primarily dependent on imaging studies. For patients with acute cerebral hemorrhage, CTA is a rapid and effective method to correctly diagnose MMD $(21,22)$, although examination by DSA may provide more accurate results. The case reported in the present study was accurately diagnosed as MMD and MSIH by CTA following the onset, while laboratory tests ruled out hematologic diseases.

The treatment of MSIH depends on the volume and location of the hematoma. However, treatment differs from that for single intracranial hemorrhage, primarily because multiple hemorrhages can be scattered, and not all multiple hemorrhages can be removed at the same time. For instance, in the current study, only the hematomas in the left basal ganglia and the posterior temporal lobe were removed, while the hemorrhage in the frontal lobe was not treated as it was risky and unnecessary, although simultaneous decompressive craniectomy was performed. The efficacy of MSIH treatment was not better over that for a single hemorrhage (23). The study by Laiwattana et al (3) reported that the prognosis for MSIH was 
poor, particularly for cases involving the bilateral thalamus. The case reported in the current study involved only the hemisphere on the left side of the meninges. Compared with MSIH cases involving both hemispheres, the efficacy following active treatment was relatively satisfactory in the current case.

Therefore, considering the present report of MMD-induced MSIH, we suggest that the possibility of MMD should be considered when an MSIH case is identified. In clinical practice, MMD can be accurately diagnosed using CTA and may lead to pathological changes in small arteries, causing them to become fragible. Rupture and hemorrhage occurring at one site may induce a transient increase in blood pressure, causing the rupture of small arteries at other sites, and thus leading to MSIH. Subsequent to ruling out disorders of the blood and blood coagulation, hematoma evacuation and decompression should be performed in selective cases, which can lead to good prognosis.

In conclusion, MSIH caused by MMD is extremely rare; microbleedings are typical of MSIH, and severe MSIH rarely occurs. The cause of severe MSIH may be a result of transient hypertension following a hemorrhage, and the hypertension can result in simultaneous hemorrhages in other locations. In the present study, the treatment of MSIH was difficult, primarily because hemotomas were scattered and damaged numerous functional regions of the brain. Timely decompressive surgery and hemotoma removal is vital in the treatment of MSIH.

\section{References}

1. Hayashi K, Morofuji Y, Horie N and Izumo T: A case of neurofibromatosis type 1 complicated with repeated intracerebral hemorrhage due to quasi-moyamoya disease. J Stroke Cerebrovasc Dis 24: e109-e113, 2015.

2. McCormick WF and Rosenfield DB: Massive brain hemorrhage: A review of 144 cases and an examination of their causes. Stroke 4: 946-954, 1973.

3. Laiwattana D, Sangsawang B and Sangsawang N: Primary multiple simultaneous intracerebral hemorrhages between 1950 and 2013: Analysis of data on age, sex and outcome. Cerebrovasc Dis Extra 4: 102-114, 2014.

4. Finelli PF: A diagnostic approach to multiple simultaneous intracerebral hemorrhages. Neurocrit Care 4: 267-271, 2006.

5. Kikuta K, Takagi Y, Nozaki K, Sawamoto N, Fukuyama H and Hashimoto N: The presence of multiple microbleeds as a predictor of subsequent cerebral hemorrhage in patients with moyamoya disease. Neurosurgery 62: 104-111; discussion 111-122, 2008.

6. Kim T, Oh CW, Bang JS, Kim JE and Cho WS: Moyamoya disease: Treatment and outcomes. J Stroke 18: 21-30, 2016.
7. Kim JS: Moyamoya disease: Epidemiology, clinical features, and aiagnosis. J Stroke 18: 2-11, 2016.

8. Balestreri M, Czosnyka M, Chatfield DA, Steiner LA, Schmidt EA, Smielewski P, Matta B and Pickard JD: Predictive value of Glasgow Coma Scale after brain trauma: Change in trend over the past ten years. J Neurol Neurosurg Psychiatry. 75: 161-162, 2004.

9. Seeder L: Muscle strength grading. Ann Emerg Med 12: 407, 1983.

10. Stemer A, Ouyang B, Lee VH and Prabhakaran S: Prevalence and risk factors for multiple simultaneous intracerebral hemorrhages. Cerebrovasc Dis 30: 302-307, 2010.

11. Chanda A and Nanda A: Multiple cavernomas of brain presenting with simultaneous hemorrhage in two lesions: A case report. Surg Neurol 57: 340-344; discussion 334-335, 2002.

12. Kidd D, Plant GT, Scaravilli F, McCartney AC, Stanford M and Graham EM: Metastatic choriocarcinoma presenting as multiple intracerebral haemorrhages: The role of imaging in the elucidation of the pathology. J Neurol Neurosurg Psychiatry 65: 939-941, 1998.

13. Yen CP, Lin CL, Kwan AL, Lieu AS, Hwang SL, Lin CN and Howng SL: Simultaneous multiple hypertensive intracerebral haemorrhages. Acta Neurochir (Wien) 147: 393-399; discussion 399, 2005

14. Gilles C, Brucher JM, Khoubesserian P and Vanderhaeghen JJ: Cerebral amyloid angiopathy as a cause of multiple intracerebral hemorrhages. Neurology 34: 730-735, 1984.

15. Mauriño J, Saposnik G, Lepera S, Rey RC and Sica RE: Multiple simultaneous intracerebral hemorrhages: Clinical features and outcome. Arch Neurol 58: 629-632, 2001.

16. Komiyama M, Yasui T, Tamura K, Nagata Y, Fu Y and Yagura H: Simultaneous bleeding from multiple lenticulostriate arteries in hypertensive intracerebral haemorrhage. Neuroradiology 37: 129-130, 1995.

17. Piao J, Wu W, Yang Z and Yu J: Research progress of moyamoya disease in children. Int J Med Sci 12: 566-575, 2015.

18. Hosoda Y, Ikeda E and Hirose S: Histopathological studies on spontaneous occlusion of the circle of Willis (cerebrovascular moyamoya disease). Clin Neurol Neurosurg 99 (Suppl 2): S203-S208, 1997.

19. Zhang L, Xu K, Zhang Y, Wang X and Yu J: Treatment strategies for aneurysms associated with moyamoya disease. Int J Med Sci 12: 234-242, 2015.

20. Ryan RW, Chowdhary A and Britz GW: Hemorrhage and risk of further hemorrhagic strokes following cerebral revascularization in Moyamoya disease: A review of the literature. Surg Neurol Int 3: 72, 2012.

21. Sugino T, Mikami T, Ohtaki S, Hirano T, Iihoshi S, Houkin K and Mikuni N: Assessment of moyamoya disease using multidetector row computed tomography. J Stroke Cerebrovasc Dis 22: 644-649, 2013

22. Zhang J, Wang J, Geng D, Li Y, Song D and Gu Y: Whole-brain CT perfusion and CT angiography assessment of Moyamoya disease before and after surgical revascularization: Preliminary study with 256-slice CT. PLoS One 8: e57595, 2013.

23. Chen Y, Henon H, Bombois S, Pasquier F and Cordonnier C: Multiple simultaneous spontaneous intracerebral hemorrhages: A rare entity. Cerebrovasc Dis 41: 74-9, 2016. 